Review Article

\title{
Functional Role of circRNAs in the Regulation of Fetal Development, Muscle Development, and Lactation in Livestock
}

\author{
Tianle He $\mathbb{D}^{1},{ }^{1}$ Qingyun Chen $\mathbb{D}^{1},{ }^{1}$ Ke Tian $\mathbb{D}^{1,2}$ Yinzhao Xia $\mathbb{D}^{1},{ }^{1}$ Guozhong Dong $\mathbb{D}{ }^{1}$ \\ and Zhenguo Yang iD ${ }^{1}$ \\ ${ }^{1}$ Laboratory for Bio-Feed and Molecular Nutrition, College of Animal Science and Technology, Southwest University, \\ Chongqing, China \\ ${ }^{2}$ The United Graduate School of Agricultural Science, Gifu University, Gifu, Japan
}

Correspondence should be addressed to Guozhong Dong; gzdong@swu.edu.cn and Zhenguo Yang; guoguo00002@163.com

Received 25 February 2020; Revised 23 January 2021; Accepted 5 February 2021; Published 20 February 2021

Academic Editor: Hamid Mukhtar

Copyright (c) 2021 Tianle He et al. This is an open access article distributed under the Creative Commons Attribution License, which permits unrestricted use, distribution, and reproduction in any medium, provided the original work is properly cited.

\begin{abstract}
circRNAs are a class of endogenous noncoding RNA molecules with closed loop structures. They are mainly responsible for regulating gene expression in eukaryotic cells. With the emergence of high-throughput RNA sequencing (RNA-Seq) and new types of bioinformatics tools, thousands of circRNAs have been discovered, making circRNA one of the research hotspots. Recent studies have shown that circRNAs play an important regulatory role in the growth, reproduction, and formation of livestock products. They can not only regulate mammalian fetal growth and development but also have important regulatory effects on livestock muscle development and lactation. In this review, we briefly introduce the putative biogenic pathways and regulatory functions of circRNA and highlight our understanding of circRNA and its latest advances in fetal development, muscle development, and lactation biogenesis as well as expression in livestock. This review will provide a theoretical basis for the research and development of related industries.
\end{abstract}

\section{Introduction}

Circular RNAs (circulRNAs, circRNAs) are widely found in eukaryotic cells; they are a special type of nucleotide sequences containing conserved microRNAs (miRNAs) binding sites [1]. Studies have shown that most of the circRNAs currently found are noncoding RNAs that do not encode proteins, but they play as a function at specific stages of biological tissue differentiation and development [2]. In order to explore the effects of circRNAs on fetal development, muscle development, and milk formation quality in livestock, scholars have done a lot of basic research.

On the one hand, some studies have shown that circRNAs play an important role in regulating the lactation of livestock [3-5]. Some studies detected a total of 37,818 circRNAs in goat breast tissue, of which 864 circRNAs were differentially expressed, 611 were upregulated, and 253 were down-regulated [6], so they speculated that these circRNAs may be related to breast development and lactation.
Furthermore, studies identified 4,048 circRNAs in the mammary glands of cows on the day 90 and 250 postpartum by RNA sequencing (RNA-seq), of which only 2,231 circRNAs were expressed simultaneously in both day 90 and day 250 [7], suggesting that circRNAs may have a higher stage specificity during lactation. In addition, among the 4,906 circRNAs detected in mammary gland (MG) tissues of SmallTailed Han (STH) sheep and Gansu Alpine Merino (GAM) sheep parenchyma at peak lactation, 33 circRNAs were expressed differentially, including 18 upregulated circRNAs and 15 downregulated circRNAs [8]. Five circRNAs (circ19403, circ-015111, circ-014121, circ-007220, and circ004632) were only expressed in the MG of the STH sheep and 4 circRNAs (circ-004110, circ-021253, circ-017116, and circ-003297) were only expressed in the MG of the GAM sheep [8].

On the other hand, a large number of studies have shown that circRNAs play an important role in regulating muscle development of livestock [2,9-11]. Sun et al. found that 
4,360 candidate circRNA were differentially expressed in the longissimus dorsi of Landrace and Lancang pigs. Of these, 1,401 circRNAs were upregulated in the Lantang library, while 2,959 circRNAs were downregulated. Of the differentially expressed circRNAs, 236 candidates were edited from 93 functional hosting genes related to myogenesis, inspiring us that circRNAs can play a regulatory role in animal muscle development [12]. Wang et al. showed that circTTN can activate IGF-2/phosphatidylinositol 3 kinase (PI3K)/AKT signaling pathway through competitive binding with miR-432 and promote the proliferation and differentiation of bovine primary myoblasts, and their further studies have shown that overexpression and inhibition of circTTN can jointly induce its role in promoting the proliferation and differentiation of bovine primary myoblasts [13].

In addition, studies have shown that circRNAs can regulate fetal development, and their expression is the most abundant and complex in the cerebral cortex on the 60th day of pregnancy [14-16]. Studies have shown that there are 10,032 circRNAs in human ocytes and embryos, a small portion of which are maternally expressed, and most of the circRNAs have been shown to be related to animal embryo development [17]. Our previous studies have shown that high-energy diets jointly regulate placental nutrient transport and fetal development in Yorkshire gilts through feedback between circ-Amotl1 and miR-17-5p [18]. It can be seen that circRNAs have a specific effect on fetal development. Here, we will focus on the important molecular mechanism and mode of action of circRNAs in the development of animal husbandry. The purpose of this study is to provide researchers with more circRNA-related theories to guide the development of animal husbandry.

\section{Overview of circRNAs}

2.1. Discovery and Classification of circRNAs. circRNAs were first extracted from several RNA viruses in the 1970s [19], but for a long time, they were considered to be mRNA transcription errors because of their low abundance in organisms. Subsequently, circRNAs were successively found to exist in archaea, nematodes, zebrafish, mice, and human cells and considered to do not encode proteins [19]. With the development of science and technology as well as the advent of new computational methods for nonpolyadenylated RNA transcription, circRNAs have begun to be highly valued, and circRNAs have gradually become one of the research hotspots. Recent studies have shown that circRNAs play an important role in various physiological and pathological processes and can even serve as templates for protein translation [20]. In order to carry out follow-up research in a more organized way, circRNAs could be categorized into three types, including exon circRNAs, intron circRNAs, and exon and intron mixed circRNAs based on the genomic loci and the relationship with the connected parental transcript [21-23].

2.2. The Formation of circRNAs. Recent studies have shown that circRNAs are mainly formed by reverse splicing of RNA into loops [24], and most eukaryotic circRNAs are formed by classical spliceosome or group I/II ribozymes
[24-26]. Li et al. pointed out two hypotheses for the formation of circRNAs: first, the lasso structure drives cyclization, and the lasso structure is a by-product of exon hopping. After the intron in the lasso structure is removed, exons can be connected to form circRNAs; the second is intron pairing driving cyclization, and there is an intron with reverse complementary sequence at both ends of the ring-shaped exon. The pairing mediation of the reverse complementary sequence of the intron can make the splicing donor and splice recipient of the exon spliced into a ring closer to each other in space, thus forming circRNAs [21]. Pagliarini et al. found that RNA-binding proteins can also specifically bind to flanking introns at both ends of RNA, which can reduce the distance between splice and donor acceptor, finally form circRNAs [27, 28] (see Figure 1). In addition, CDR1as can also be generated by mammalian-wide interspersed repeats (MIRs) in mammals [29]. These findings suggest that the formation of circRNAs may be affected by many other factors. Therefore, more research is needed to fully understand the formation of circRNAs.

2.3. Characteristics of circRNAs and Their Biological Functions. According to the research results in recent years, the characteristics of circRNAs can be summarized as follows:

(1) Localization specificity: most circRNAs are exonic circular RNAs (EcircRNA), which are mainly present in the cytoplasm, and only a few circular intronic RNAs (CiRNA) and exon-intron circular RNAs (EIciRNA) containing introns exist in the nucleus of eukaryotic cells [30].

(2) Expression specificity: medical studies have shown that circRNAs have certain tissue and disease specificity [30].

(3) Coding specificity: noncoding RNA can only play a regulatory role during transcription or post-transcription without coding protein formation [1], but a few can encode proteins $[31,32]$.

(4) Structural stability: circRNAs do not have $5^{\prime}$ and caps and $3^{\prime}$ end polyA tails and are not easily degraded by exonuclease RNAseR [33, 34].

(5) circRNAs: they are highly conserved in many species, such as humans, mice, nematodes, and zebrafish [21], and only a small number of them can rapidly evolve and change [34].

(6) Abundance and diversity: only circRNAs found in eukaryotic cells have exceeded 20,000 species [1], a few are formed by direct cyclization of introns, and most of them come from exons [35].

Confirmed or speculated through research, circRNAs control gene expression at different levels, including transcription, pre-mRNA splicing, mRNA translation, and protein function. In recent years, more researches have focused on that circRNAs can act as sponges for miRNAs. When circRNAs specifically bind to multiple miRNAs, they can 


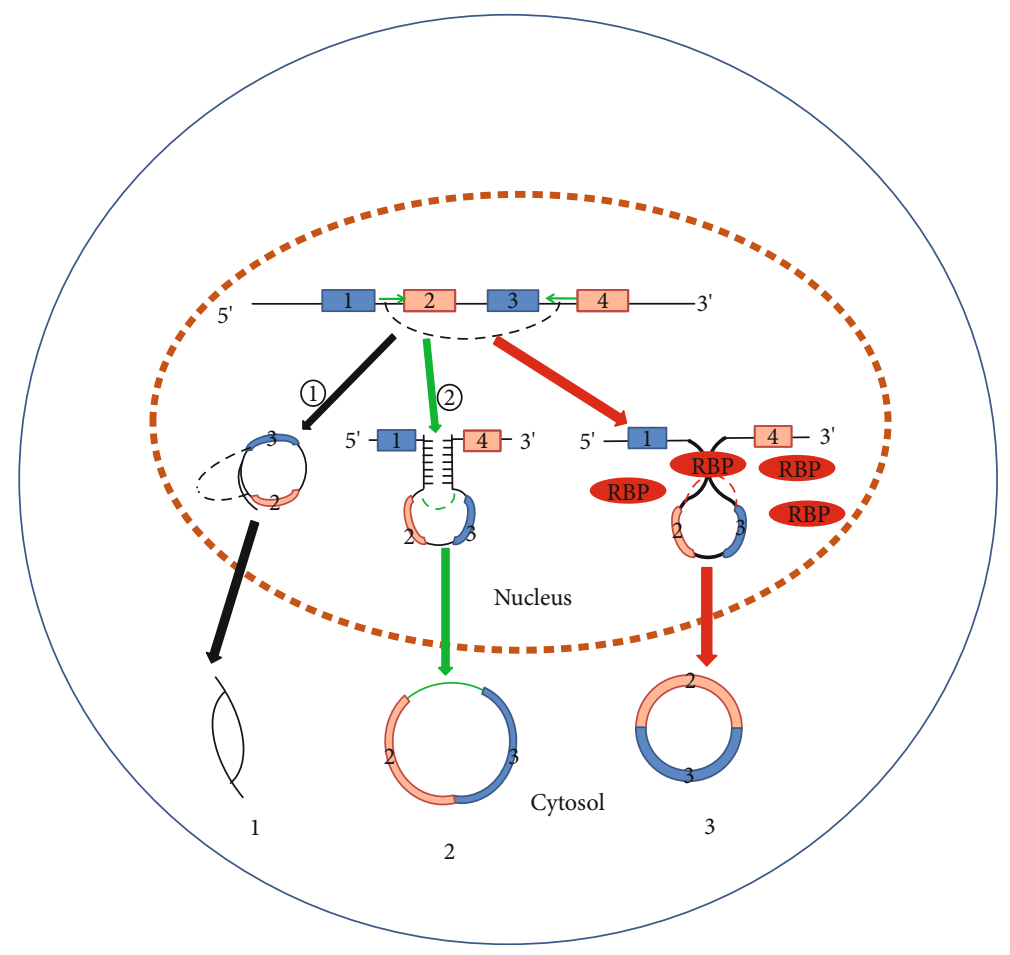

FIgURE 1: The main formation of circRNAs: 1: circRNAs are formed after introns in the lasso structure are excised; 2: intron pairing driving cyclization, thus forming circRNAs; 3: the RNA-binding protein specifically binds to flanking introns at both ends of the RNA, thus forming circRNAs.

release the inhibitory effect of miRNAs on their targeted mRNAs, thereby promoting the transcription and protein translation of targeted genes [36-41]. It has also been reported that circRNAs can be spliced with linear RNA, thereby smoothly "capturing" mRNA and then regulating the life process [42]; circRNAs can also interact with RNA polymerase II (Pol II), thereby promoting the transcription of parent genes [31]. Some circRNAs can regulate the normal physiological activities of biological individuals by participating in the synthesis of body proteins $[43,44]$. For example, circ-Amotl1 physically binds to both 3-phosphoinositidedependent protein kinase 1 (PDK1) and protein kinase $B$ (AKT), facilitating the cardioprotective nuclear translocation of pAKT [45]. Very few circRNAs can be used as translation templates to promote translation [44]. Recently, some studies have shown that circRNAs can also be used as biomarker molecules [46, 47] and are currently used as markers for the diagnosis of related diseases [45].

2.4. circRNAs Regulate Fetal Development in Livestock. Mammalian fetal development refers to the process by which fertilized eggs develop into larvae, and the fetus is regulated by many factors during early development. Under the joint control of multiple factors, fertilized eggs can successfully divide and differentiate into normal fetuses. In the production of animal husbandry, healthy livestock fetuses are the foundation of population continuity and industrial development. The higher the number and health of livestock fetuses, the greater the value of breeding and economic value. Therefore, research on regulating fetal development in mammals is essential. Some scholars have found through deep RNA sequencing and bioinformatics analysis that the expression of circRNAs has tissue-cell specificity and stage-specific developmental differentiation during the embryonic stage of animals $[48,49]$. Many recent studies have shown that circRNAs may have unique and important functions during embryonic development, and related studies on the longterm development of the fetus after birth are gradually increasing with the placenta-based microenvironment of the fetal interface [49]. The researchers found that circRNAs play an important role in a variety of biological processes, including cell differentiation and development, and mammalian pregnancy is accompanied by cell differentiation and development of individual fetuses, which indicates that circRNAs are very likely to growth and development $[19,50]$. Dang et al. studied human embryonic development and found that there were 1,032 types of circRNAs in the exon region of 2,974 genes in cells (endocell clusters, nourishing ectoderm cells, etc.) at different developmental stages [15]. Most circRNAs can perform specific dynamic regulation during embryonic development and are mainly involved in the regulation of material metabolic pathways in early embryonic development [15]. The development of a mammalian fetal brain is a crucial step for embryonic development. Szabo et al. have pointed out in the study of human fetal brain development that circRNAs in some areas of the fetal brain (such as the frontal cortex) are significantly enriched and these circRNAs are important for studying human embryonic development [48]. Wang et al. selected 5 brain tissues from 6 time points of fetal pig development to 
detect the expression of circRNAs [14]. They found that the sow's quantity and complexity of circRNAs expression were most obvious in the cortex at the 60th day of pregnancy of the 13,854 expressed genes detected; 2,195 genes expressed 4,634 unique circRNAs; In addition, Hirotsu et al. found that the expression of circRIMS2 in the embryonic pig cortex reached a peak during development, suggesting that circRNAs are one of the major factors regulating mammalian embryonic brain development [51]. Shen et al. used adult intrauterine growth restriction (IUGR) pigs and normal pigs as models and used RNA-Seq technology to detect the expression of circRNAs in the liver during pregnancy [52]. A total of 403 circRNAs were found, of which 44 had differences in expression $(p<0.05)$; their study showed people a variety of circRNAs that may be involved in the development of IUGR pigs, laying a foundation for future studies of fetal development during pregnancy [52]. Fan et al. sequenced 913 new linear transcripts and 2,891 circRNAs found in mouse preimplantation embryos by SUPeR-seq, analyzed the abundance of circRNAs, the function of enriched genes, and the characteristics of circRNAs sequence during development, and speculated that these circRNAs may play a role in the embryonic development of other animals [53]. Zhang et al. constructed circRNA miRNA during the implantation period of dairy goat embryos and found that circRNA8073 (cir8073) can reduce the level of miR-181a by acting as a miRNA sponge [54]. This effect indirectly increases the expression of neurotensin in endometrial epithelial cells (EECS), thereby inhibiting the apoptosis of EECS during embryo implantation, which is conducive to embryo implantation and embryo development. In addition, $\mathrm{Xu}$ et al. speculated that the tissue-specific and developmental specificity of circRNAs in adults and fetuses may be determined by different expression levels of circRNAs in tissues after a large number of studies, but there has been no new breakthrough in this area [55]. Other studies have reported that circRNAs also play important roles in gametogenesis and preeclampsia $[56,57]$. Studies in pigs have also shown that circRNAs are expressed at different levels in fetuses with different body weights and attachment sites [16]. Researchers showed that many miRNAs in animals can be preferentially expressed in the placenta and are related to pregnancy and birth circRNAs are directly related to the regulation of miRNAs expression [58]. Therefore, the researchers speculated that circRNAs and miRNAs were affected during the placental nutrient transport comprehensive regulatory role. In fact, as early as 2013, Hansen found that miRNAs play important regulatory functions in animal embryonic development [36]. Maternal high energy diet during pregnancy could promote placental nutrient transport and accelerate fetal intrauterine growth in the third trimester of pregnancy [18]; the study of sows finally confirmed our conjecture; we measured the expression of circ-Amotl1 and miR-17-5p in the placenta of sows on the 90th day of pregnancy. The results showed that the expression level of circ-Amotl1 in placenta at the end of uterine horn in the low energy group and high energy group was significantly higher than that in the cervix, while the expression level of miR-17-5p was significantly decreased in the low energy group and high energy group [18]. Finally, we found that the uniform distribution of nutrition and fetal development at different placental attachment sites were affected by circ-Amotll and miR-17-5p; it is suggested that the interaction between circ-Amotl1 and miR-17-5p can regulate the ability of maternal placental nutrient transport and then regulate embryonic development [18]. The results of this series of studies have inspired us that circRNAs are likely to have an important effect on embryonic development through the sponge effect of miRNAs. The regulation of circRNAs on mammalian embryonic development is also becoming more and more obvious, but there are few studies on domestic animals. Therefore, scientific researchers need to make persistent efforts based on previous research.

2.5. Regulation of circRNAs on Muscle Development in Livestock. Animal meat is rich in protein, with a complete range of essential amino acids, and the proportion of each amino acid is appropriate, which is close to the protein composition of the human body. It has been reported that circRNAs can play a pivotal role in muscle development through specific miRNAs. Researchers have found that the number of circRNAs in animal skeletal muscle and myoblasts ranges from 2,000 to 37,000 [59-65]. Cao et al. identified circRNAs of the longest muscle in sheep and found that many circRNAs such as circ776 can interact with specific miRNAs for muscle growth and development [66]. After further research, they found that the host genes of circRNAs are involved in muscle cell development and related signaling pathways. Liang et al. comprehensively analyzed circRNAs in three skeletal muscles of Guizhou mini pigs (S. scrofa), identified 149 circRNAs that may be related to muscle growth, and found that their host genes were significantly involved in muscle development, contraction, chromatin modification, cationic homeostasis, and ATP hydrolysis coupled proton transport [62]. After deep research, they found ssc-ciR-02753, ssc-ciR-04353, ssc-ciR-04335, ssc-ciR04349, ssc-ciR-04348, ssc-ciR-04359, ssc-ciR-03066, sscciR-03069, and ssc-ciR-03065 can regulate muscle growth by affecting cell proliferation and fusion during early postpartum muscle development [62]. The researchers also pointed out that circLMO7 can bind to miR-378a-3p to cause HDAC4 to increase and MEF2A expression to decrease and ultimately promote bovine myoblast differentiation and survival [63]. Peng et al. found that the expression level of circSNX29 in bovine embryonic skeletal muscle was significantly higher than that of adult skeletal muscle [67]. The overexpression of circSNX29 can inhibit myoblast proliferation and promote myoblast differentiation. Further studies have shown that circSNX29 can bind to its target miR-744 and keep miR-744 away from Wnt5a, resulting in increasing mRNA expression level of Wnt5a and phosphorylation level of protein kinase $\mathrm{C}(\mathrm{PKC})$, thus effectively activating Wnt5a/Ca ${ }^{2+}$ signal pathway and regulating embryonic bovine muscle development. Other studies have shown that circFUT10 produced by FUT10 is mainly expressed in bovine skeletal muscle tissue. When circFUT10 is overexpressed, it can inhibit cell proliferation, induce myoblast apoptosis, and ultimately promote myoblast differentiation [68]. $\mathrm{Li}$ et al. pointed out that circFGFR4 is highly expressed in 
bovine skeletal muscle and overexpression of circFGFR4 can also cause apoptosis, thereby promoting myoblast differentiation [69]. RNAhybrid and TargetScan showed that circFGFR4 contains 18 putative miR-107 binding sites, and luciferase experiments and RNA pull-down confirmed the interaction between miR-107 and circFGFR4, and they found that Wnt3a was miR-107 target, while Wnt3a can inhibit myotube formation and protect myoblasts from apoptosis. However, whether this is a major feature of Wnt3a remains to be verified. Based on the above studies, Siengdee et al. pointed out that miR-194 can be upregulated during porcine $\mathrm{C} 2 \mathrm{C} 12$ myoblast differentiation [70]. Although studies have shown that miR-194-5p and circZfp609 (circRNA) have four binding sites, when circZfp609 binds to miR-194-5p, it can inhibit BCLAF1 (Bcl2-associated transcription factor 1) [71], which can inhibit further differentiation of muscle cells. However, there are no reports on whether the combination of circZfp609 and miR-194-5p can affect the differentiation of livestock myoblasts. Thus, it can be seen that circRNAs can regulate the muscle development of livestock through CeRNA (circRNA-miRNA-mRNA) coexpression network (see Figure 2).

Many studies have shown that animal muscle formation is regulated by RNA-binding proteins and long-chain noncoding RNA (ncRNA) at the posttranscriptional level [72, 73]. Circ-ZNF609 can initiate translation and directly participate in muscle cell development through a mechanism independent of cap structure [74]. Pandey et al. [75] indicated that binding of Pur protein to CircSAMD4 can promote muscle development by reducing $\mathrm{MHC}$ transcription. Pura and Purb are myogenic inhibitors that inhibit transcription of the myosin heavy chain (MHC) protein family. Silencing of circsamD4 enhances the binding of the PUR protein to the MHC promoter, while overexpression of circsamD4 interferes with the binding of the PUR protein to the MHC promoter, suggesting that circsamD4 can bind to the PUR protein and prevent its interaction with DNA. These effects were cancelled when the mutant circSamd4 without the PUR binding site was used. Yin et al. [76] found that circFAM188B contains an open reading frame (ORF), which can be directly translated into circFAM188B-103aa and thus directly promote the development of skeletal muscle in broilers. Thus, it can be seen that circRNAs can also regulate the muscle development of livestock and poultry at the transcriptional level.

circRNAs are one of the more comprehensive studies of ncRNAs, which are particularly outstanding in regulating animal muscle development. The above series of research results inspired us that circRNAs are involved in the regulation of muscle development. Although some of the research on the regulation mechanism is not clear, in general, most of the current studies on circRNAs to regulate muscle formation have turned to the research of related miRNAs. We find that many researchers are trying to explain the related physiological phenomena by studying the interaction between circRNAs and miRNAs, but the specific binding of circRNAs and miRNAs is only a small part of the regulation of life activities by circRNAs (see Figure 2).

2.6. Regulation of circRNAs on Lactation and Milk Quality in Livestock. In recent years, a large number of studies have shown that circRNAs play an important role in regulating the lactation of livestock $[5,8,77,78]$. Lin et al. found that the expression of miR-103 was related to lactation through high-throughput sequencing [79]. Further functional analysis showed that the overexpression of miR-103 in mammary epithelial cells increased the transcription of genes related to milk fat synthesis, resulting in the formation of fat droplets, the accumulation of triglycerides, and the upregulation of the proportion of unsaturated fatty acids [79]. In addition, studies have shown that miR-103 is corelated with one upregulated circRNA (circRNA_007873) and two downregulated circRNAs (circRNA_010763, cir-cRNA_015622) at the same time [80]. However, there is still a lack of direct evidence that circRNAs and miR-103 work together to regulate goat lactation. Zhang et al. found that circRNAs are abundant in the mammary glands of cows, and the expression of circRNAs in the mammary glands of cows is different at different lactation stages [7]; they reported that the content and specificity of circRNAs in bovine mammary glands at 90 and 250 days postpartum were different and specific. The 90 -day and 250-day periods corresponded to the prelactation period and the postlactation period, respectively. The comparison showed that various nutrient contents of dairy cows changed significantly during the two lactation periods. It is well known that casein is an important indicator of milk quality assessment, also a major protein in mammals including cows, sheep, and human milk, and an important source of amino acids and calcium and phosphorus for young children [81]. Zhang et al. found four casein-binding genes in milk, namely, $\alpha$ s1-casein gene (CSN1S1), $\alpha$ s2-casein gene (CSN1S2), casein $\beta$ gene (CSN2), and casein $\mathrm{k}$ gene (CSN3), can produce circRNAs in bovine mammary glands, and the abundance of circRNAs expressed by CSN1S1 and CSN2 on the 90th day of lactation was significantly higher than that on the 250th day of lactation. Interestingly, the abundance of circRNAs is consistent with the change in the proportion of casein in milk during lactation [7]. The above study inspired us that circRNAs have an important relationship with milk production and milk quality in dairy cows. Combining the discovery that the miR-2284 family is expressed at high levels in bovine mammary epithelial cells [82], Zhang et al. pointed out that bovine casein circRNAs have high-density binding sites for the miR-2284 family and they also predicted miR-2284 targets; these are mRNAs of CSN1S1 and CSN2, and casein circRNAs may act as miR-2284 sponges to regulate casein translation [7]. In addition, $\mathrm{Ma}$ et al. sequenced lactating (early and mature) goat breast tissues and found that circRNAs can form ceRNAs (circRNA-miRNA-mRNA) together with mRNA and miRNAs to participate in the regulation of goat lactation [6]. That is to say, circRNA establishes a functioning system related to mRNA through the intermediate targeting of miRNA, thereby forming a network system in which circRNAs regulate the translation process of mRNA. Therefore, the ceRNAs (circRNA-miRNA-mRNA) established by $\mathrm{Ma}$ et al. based on previous studies provide a new research idea for analyzing the specific regulation of a gene in related biological processes [6]. This idea currently has an irreplaceable role in studying circRNAs as miRNA sponges. Bian et al. found that miR-29s regulates DNA 


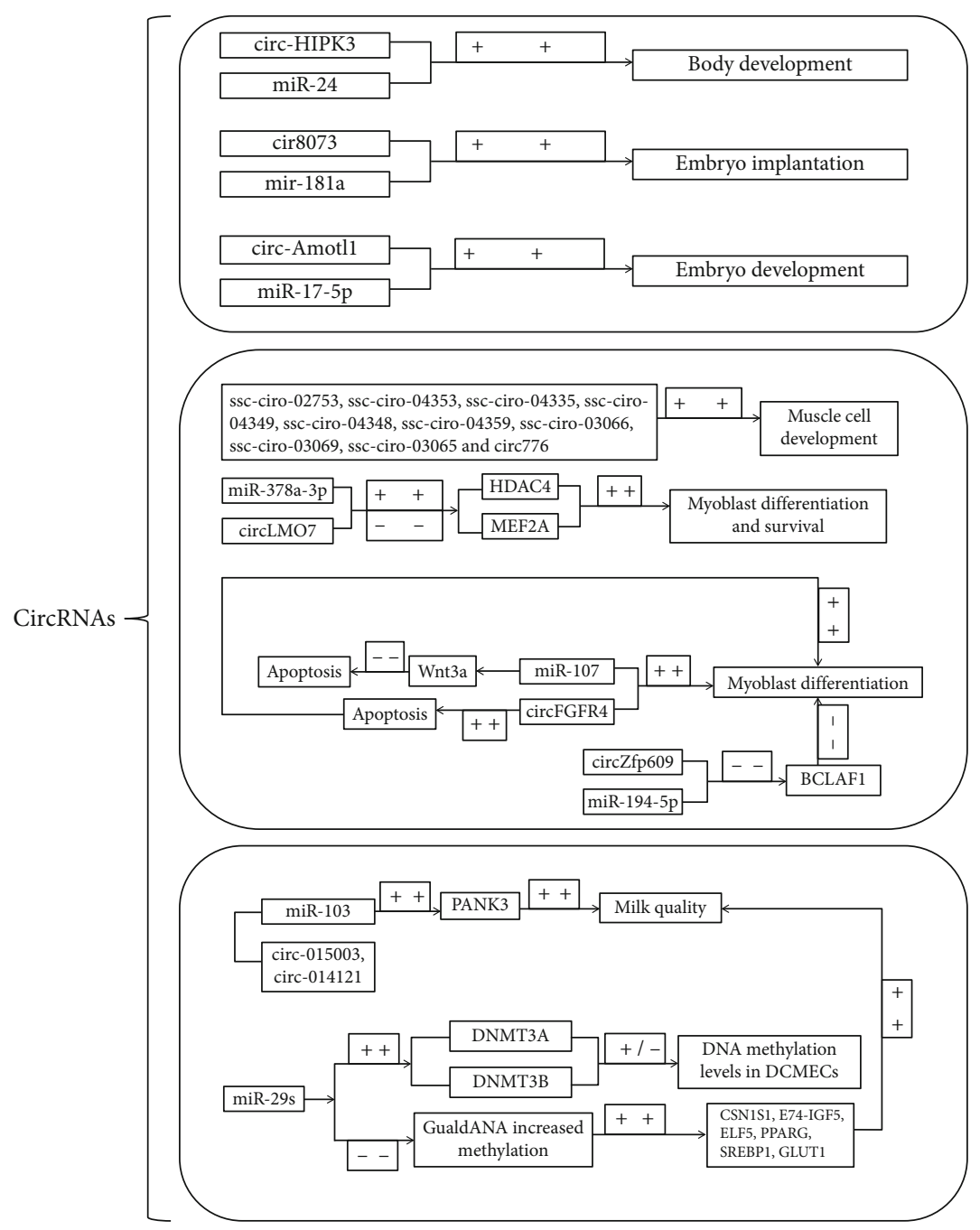

Figure 2: “+ +" means promotion, “- -" means inhibition, and the arrow points to the regulation direction.

methylation levels in DCMECs by reverse targeting DNMT3A and DNMT3B, and the inhibitory effect of miR29 will cause GualdANA hypermethylation, increase methylation level, and promote important lactation related genes (casein phosphatase 1 (CSN1S1), E74-IGF5 (ELF5), peroxisome proliferator-activated receptor gamma (PPARG), sterol regulatory element binding protein-1(SREBP1), and glucose transporter 1 (GLUT1)) and further regulate the secretion of milk proteins, triglycerides, and lactose in dairy mammary epithelial cells [83]. Wright et al. also found that many miRNAs are involved in the regulation of milk protein synthesis and mammary gland development in dairy cows [84]. Through the above studies, we found that circRNAs play an important role in regulating milk production and milk quality in livestock. Combining with the ceRNA system established by $\mathrm{Ma}$ and others [6], we can speculate that the lactation process of cows similar to those regulated by mir29s will be affected by corresponding circRNAs, and Hao et al. analyzed the constructed miRNA-circRNA network and found that 43 miRNAs related to mammary gland development and lactation have a targeting relationship with circ001091 [8]. Network analysis also shows that circ-014121 and circ001091 have miR-29a and miR-29b targets, and this finding just confirms our conjecture.

To date, little research has been done on circRNAs in breast tissue. Especially in related species, the expression profile of circRNAs has only been reported in cows and goats. However, the above research results are sufficient to show that circRNAs have important regulatory effects on animal milk production, milk protein synthesis, and mammary gland development. This lays a foundation for us to explore the regulation of milk formation and milk quality by circRNAs. However, the related pathways of circRNAs to regulate milk formation and milk quality are not very clear, so exploring this series of laws has become one of the issues that need to be solved in the development of animal husbandry.

\section{Conclusions}

The discovery of circRNAs has greatly broadened our understanding of gene expression regulatory mechanisms. With the development of science and technology and the unremitting efforts of researchers, we will know more and more about circRNAs. As a small molecular substance with 
biological regulatory functions widely exists in organisms, circRNAs will become a hot area in RNA regulatory networks. In recent years, more and more evidence shows that circRNAs can regulate fetal development, muscle development, and lactation of livestock to some extent. Through the study of circRNAs, researchers can reunderstand and "manipulate" livestock fetal development, muscle development, and lactation at the genetic level, which can help improve livestock production and reproduction performance. However, at present, we are not very clear about the regulatory mechanism of circRNAs, and even some of these regulatory pathways remain only at the guessing stage. Therefore, researchers need to strengthen the research in this area to enrich the regulation theory of animal product formation and growth and development and lay the foundation for the development of agricultural economy.

\section{Data Availability}

Not applicable.

\section{Conflicts of Interest}

The authors declare that there are no conflicts of interest regarding the publication of this paper.

\section{Authors' Contributions}

Tianle He and Ke Tian contributed equally to this work.

\section{Acknowledgments}

This study was supported by the Chongqing Natural Science Foundation (Basic Research and Frontier Exploration Special Project) General Project (Grant No. cstc2019jcyj-msxmX0524), the National Natural Science Foundation of China (Grant No. 31902167), the National Key Research and Development Plan of China (Grant No. 2018YFD0501004), the National Science Foundation for Post-Doctoral Scientists of China (Grant No. 2018M640895), the Special Funding for Postdoctoral Research Projects in Chongqing (Grant No. XMT 2081061), the Excellent Youth Exchange Program of China Association for Science and Technology ([2019]293-98), and the Chongqing Innovation Funding Projects for Returned Scholars (Grant No. cx2019093).

\section{References}

[1] J. Westholm, "Genome-wide analysis of_Drosophila_circular RNAs reveals their structural and sequence properties and age-dependent neural accumulation," Cell Reports, vol. 9, no. 5, pp. 1966-1980, 2014.

[2] A. Das, A. Das, D. Das, K. Abdelmohsen, and A. C. Panda, "Circular RNAs in myogenesis," Biochimica et Biophysica Acta (BBA) - Gene Regulatory Mechanisms, vol. 1863, pp. 194372194378, 2020.

[3] C. Zhu, Y. Jiang, J. Zhu et al., "CircRNA8220 sponges MiR8516 to regulate cell viability and milk synthesis via Ras/ME$\mathrm{K} / \mathrm{ERK}$ and PI3K/AKT/mTOR pathways in goat mammary epithelial cells," Animals, vol. 10, no. 8, pp. 1347-1365, 2020.
[4] D. Wang, Z. Chen, X. Zhuang et al., "Identification of circRNA-associated-ceRNA networks involved in milk fat metabolism under heat stress," International Journal of Molecular Sciences, vol. 21, no. 11, p. 4162, 2020.

[5] B. Zeng, T. Chen, J. Luo et al., "Exploration of long non-coding RNAs and circular RNAs in porcine milk exosomes," Frontiers in Genetics, vol. 11, pp. 652-663, 2020.

[6] D. Ma, Y. Zhao, S. Yu et al., "CircRNA as CeRNA mediated by microRNA may be involved in goat lactation," Small Ruminant Research, vol. 171, pp. 63-72, 2019.

[7] C. Zhang, H. Wu, Y. Wang et al., "Circular RNA of cattle casein genes are highly expressed in bovine mammary gland," Journal of Dairy Science, vol. 99, no. 6, pp. 4750-4760, 2016.

[8] Z. Hao, H. Zhou, J. G. H. Hickford et al., "Identification and characterization of circular RNA in lactating mammary glands from two breeds of sheep with different milk production profiles using RNA-Seq," Genomics, vol. 112, no. 3, pp. 21862193, 2020.

[9] B. Yue, J. Wang, W. Ru et al., "The circular RNA circ _HUWE1_ sponges the miR-29b-_AKT3_ axis to regulate myoblast development," Molecular Therapy-Nucleic Acids, vol. 19, pp. 1086-1097, 2020.

[10] X. Yan, Z. Zhang, Y. Meng, H. Li, and J. Zhang, "Genome-wide identification and analysis of circular RNAs differentially expressed in the longissimus dorsi between Kazakh cattle and Xinjiang brown cattle," Peer J, vol. 8, pp. e8646-e8662, 2020.

[11] B. Li, D. Yin, P. Li et al., "Profiling and functional analysis of circular RNAs in porcine fast and slow muscles," Frontiers in Cell and Developmental Biology, vol. 8, pp. 322-324, 2020.

[12] J. Sun, M. Xie, Z. Huang et al., "Integrated analysis of noncoding RNA and mRNA expression profiles of 2 pig breeds differing in muscle traits," Journal of Animal Science, vol. 95, no. 3, pp. 1092-1103, 2017.

[13] X. Wang, X. Cao, D. Dong et al., "Circular RNA TTN acts as a miR-432 sponge to facilitate proliferation and differentiation of myoblasts via the IGF2/PI3K/AKT signaling pathway," Molecular Therapy-Nucleic Acids, vol. 18, pp. 966-980, 2019.

[14] D. Wang, Q. Na, G. Song, Y. Wang, and Y. Wang, "The role of circRNA-SETD2/miR-519a/PTEN axis in fetal birth weight through regulating trophoblast proliferation," BioMed Research International, vol. 2020, Article ID 9809632, 11 pages, 2020.

[15] S. Wang, X. Tang, L. Qin et al., "Integrative analysis extracts a core ceRNA network of the fetal hippocampus with Down syndrome," Frontiers in Genetics, vol. 11, pp. 565955565971, 2020.

[16] M. T. Venø, T. B. Hansen, S. T. Venø et al., "Spatio-temporal regulation of circular RNA expression during porcine embryonic brain development," Genome Biology, vol. 16, no. 1, pp. 245-262, 2015.

[17] Y. Dang, L. Yan, B. Hu et al., "Tracing the expression of circular RNAs in human pre-implantation embryos," Genome Biology, vol. 17, no. 1, pp. 130-144, 2016.

[18] Z. G. Yang, Effects of dietary energy levels on placental and fetal development in gilts and the mechanism investigation of CircAmotl1 expression, Sichuan Agricultural University, Chengdu, 2016.

[19] M. Hsu and M. Coca-Prados, "Electron microscopic evidence for the circular form of RNA in the cytoplasm of eukaryotic cells," Nature, vol. 280, no. 5720, pp. 339-340, 1979. 
[20] Y. WANG and Z. WANG, "Efficient backsplicing produces translatable circular mRNAs," RNA, vol. 21 , no. 2, pp. 172179, 2015.

[21] X. Li, S. Liu, L. Zhang et al., "A unified mechanism for intron and exon definition and back-splicing," Nature, vol. 573, no. 7774, pp. 375-380, 2019.

[22] S. Memczak, M. Jens, A. Elefsinioti et al., "Circular RNAs are a large class of animal RNAs with regulatory potency," Nature, vol. 495, no. 7441, pp. 333-338, 2013.

[23] K. Przybylak, J. C. Madden, M. T. D. Cronin, and M. Hewitt, "Assessing toxicological data quality: basic principles, existing schemes and current limitations," SAR and QSAR in Environmental Research, vol. 23, no. 5-6, pp. 435-459, 2012.

[24] Q. VICENS and E. WESTHOF, "Biogenesis of circular RNAs," Cell, vol. 159, no. 1, pp. 13-14, 2014.

[25] E. LASDA and R. PARKER, "Circular RNAs: diversity of form and function," RNA, vol. 20, no. 12, pp. 1829-1842, 2014.

[26] L. Chen, "Regulation of circRNA biogenesis," RNA Biology, vol. 12, no. 4, pp. 381-388, 2015.

[27] V. Pagliarini, A. Jolly, P. Bielli, V. di Rosa, P. de la Grange, and C. Sette, "Sam68 binds Alu-rich introns in SMN and promotes pre-mRNA circularization," Nucleic Acids Research, vol. 48, no. 2, pp. 633-645, 2020.

[28] W. Zhao, Y. Cui, L. Liu et al., "Splicing factor derived circular RNA circUHRF1 accelerates oral squamous cell carcinoma tumorigenesis via feedback loop," Cell Death \& Differentiation, vol. 27, no. 3, pp. 919-933, 2020.

[29] R. Yoshimoto, K. Rahimi, T. Hansen, J. Kjems, and A. Mayeda, "Biosynthesis of circular RNA ciRS-7/CDRlas is mediated by mammalian-wide interspersed repeats (MIRs)," iScience, vol. 23, no. 7, pp. 101345-101370, 2020.

[30] D. Li, Z. Li, Y. Yang et al., "Circular RNAs as biomarkers and therapeutic targets in environmental chemical exposure-related diseases," Environmental Research, vol. 180, pp. 108825108833, 2020.

[31] H. Chen, Y. Liu, P. Li, and D. Zhu, "RE: novel role of FBXW7 circular RNA in repressing glioma tumorigenesis," JNCI: Journal of the National Cancer Institute, vol. 111, no. 4, pp. 435446, 2019.

[32] N. Pamudurti, "Translation of CircRNAs," Molecular Cell, vol. 66, no. 1, pp. 9-21.e7, 2017, e7.

[33] H. Suzuki, Y. Zuo, J. Wang, M. Q. Zhang, A. Malhotra, and A. Mayeda, "Characterization of RNase R-digested cellular RNA source that consists of lariat and circular RNAs from pre-mRNA splicing," Nucleic Acids Research, vol. 34, no. 8, pp. e63-e69, 2006.

[34] H. SUZUKI and T. TSUKAHARA, "A view of pre-mRNA splicing from RNase R resistant RNAs," International Journal of Molecular Sciences, vol. 15, no. 6, pp. 9331-9342, 2014.

[35] X. Liu, Z. Hu, J. Zhou et al., "Interior circular RNA," RNA Biology, vol. 17, no. 1, pp. 87-97, 2020.

[36] T. Hansen, "Natural RNA circles function as efficient microRNA sponges," Nature, vol. 495, no. 7441, pp. 384-388, 2013.

[37] G. Li, M. Huang, Y. Cai, Y. Yang, X. Sun, and Y. Ke, "CircU2AF1 promotes human glioma via derepressing neurooncological ventral antigen 2 by sponging hsa-miR-7-5p," Journal of Cellular Physiology, vol. 234, no. 6, pp. 9144-9155, 2018.

[38] Q. Zheng, C. Bao, W. Guo et al., "Circular RNA profiling reveals an abundant circHIPK3 that regulates cell growth by sponging multiple miRNAs," Nature Communications, vol. 7, no. 1, pp. 11215-11227, 2016.

[39] D. Han, J. Li, H. Wang et al., "Circular RNA circMTO1 acts as the sponge of microRNA-9 to suppress hepatocellular carcinoma progression," Hepatology, vol. 66, no. 4, pp. 11511164, 2017.

[40] I. Hall, M. Climent, M. Quintavalle et al., "Circ_Lrp6, a circular RNA enriched in vascular smooth muscle cells, acts as a sponge regulating miRNA-145 function," Circulation Research, vol. 124, no. 4, pp. 498-510, 2019.

[41] K. Wang, B. Long, F. Liu et al., "A circular RNA protects the heart from pathological hypertrophy and heart failure by targeting miR-223," European Heart Journal, vol. 37, no. 33, pp. 2602-2611, 2016.

[42] R. Ashwal-Fluss, M. Meyer, N. R. Pamudurti et al., "circRNA biogenesis competes with pre-mRNA splicing," Molecular Cell, vol. 56, no. 1, pp. 55-66, 2014.

[43] W. W. du, W. Yang, X. Li et al., "A circular RNA circ-DNMT1 enhances breast cancer progression by activating autophagy," Oncogene, vol. 37, no. 44, pp. 5829-5842, 2018.

[44] L. Chen, L. Wang, Y. Ren et al., "The circular RNA circ-ERBIN promotes growth and metastasis of colorectal cancer by miR125a-5p and miR-138-5p/4EBP-1 mediated cap-independent HIF-1 $\alpha$ translation," Molecular Cancer, vol. 19, no. 1, pp. $1-$ 16, 2020.

[45] Q. Luo, X. Li, B. Fu et al., "Expression profile and diagnostic value of circRNAs in peripheral blood from patients with systemic lupus erythematosus," Molecular Medicine Reports, vol. 23, no. 1, pp. 1-1, 2020.

[46] B. Chen and S. Huang, "Circular RNA: an emerging noncoding RNA as a regulator and biomarker in cancer," Cancer Letters, vol. 418, pp. 41-50, 2018.

[47] A. Rybak-Wolf, C. Stottmeister, P. Glažar et al., "Circular RNAs in the mammalian brain are highly abundant, conserved, and dynamically expressed," Molecular Cell, vol. 58, no. 5, pp. 870-885, 2015.

[48] L. Szabo, R. Morey, N. J. Palpant et al., "Erratum to: statistically based splicing detection reveals neural enrichment and tissuespecific induction of circular RNA during human fetal development," Genome Biology, vol. 17, no. 1, pp. 263-265, 2016.

[49] J. ROSSANT and J. C. CROSS, "Placental development: lessons from mouse mutants," Nature Reviews Genetics, vol. 2, no. 7, pp. 538-548, 2001.

[50] A. FATICA and I. BOZZONI, "Long non-coding RNAs: new players in cell differentiation and development," Nature Reviews. Genetics, vol. 15, no. 1, pp. 7-21, 2014.

[51] A. Hirotsu, Y. Iwata, K. Tatsumi, Y. Miyai, T. Matsuyama, and T. Tanaka, "Maternal exposure to volatile anesthetics induces IL-6 in fetal brains and affects neuronal development," European Journal of Pharmacology, vol. 863, pp. 172682-172692, 2019.

[52] L. Shen, S. Zhang, Q. Li et al., "The landscape of non-coding RNA in an adult pig model of intrauterine growth restriction," Cellular Physiology and Biochemistry, vol. 50, no. 5, pp. 17641778, 2018.

[53] X. Fan, X. Zhang, X. Wu et al., "Single-cell RNA-seq transcriptome analysis of linear and circular RNAs in mouse preimplantation embryos," Genome Biology, vol. 16, no. 1, pp. 148-164, 2015.

[54] L. Zhang, X. Liu, S. Che et al., "Endometrial epithelial cell apoptosis is inhibited by a ciR8073-miR181a-neurotensis pathway 
during embryo implantation," Molecular Therapy - Nucleic Acids, vol. 14, pp. 262-273, 2019.

[55] T. Xu, J. Wu, P. Han, Z. Zhao, and X. Song, "Circular RNA expression profiles and features in human tissues: a study using RNA-seq data," BMC Genomics, vol. 18, no. S6, pp. 680-691, 2017.

[56] X. Hu, J. Ao, X. Li, H. Zhang, J. Wu, and W. Cheng, "Competing endogenous RNA expression profiling in pre-eclampsia identifies hsa_circ_0036877 as a potential novel blood biomarker for early pre-eclampsia," Clinical Epigenetics, vol. 10, no. 1, pp. 48-59, 2018.

[57] Y. Qian, Y. Lu, C. Rui, Y. Qian, M. Cai, and R. Jia, "Potential significance of circular RNA in human placental tissue for patients with preeclampsia," Cellular Physiology and Biochemistry, vol. 39, no. 4, pp. 1380-1390, 2016.

[58] T. He, G. Dong, and Z. Yang, "Regulation of circRNAs on mammalian placental nutrient transport and fetal development," Acta Veter inaria et Zootechnica Sinica, vol. 50, no. 10, pp. 1955-1962, 2019.

[59] K. Huang, M. Chen, D. Zhong et al., "Circular RNA profiling reveals an abundant circEch1 that promotes myogenesis and differentiation of bovine skeletal muscle," Journal of Agricultural and Food Chemistry, vol. 12, pp. 1520-1530, 2020.

[60] R. Liu, X. Liu, X. Bai, C. Xiao, and Y. Dong, "Identification and characterization of circRNA in longissimus dorsi of different breeds of cattle," Frontiers in Genetics, vol. 11, pp. 565085565098, 2020.

[61] C. Li, X. Li, Y. Yao et al., "Genome-wide analysis of circular RNAs in prenatal and postnatal muscle of sheep," Oncotarget, vol. 8, no. 57, pp. 97165-97177, 2017.

[62] G. Liang, Y. Yang, G. Niu, Z. Tang, and K. Li, “Genome-wide profiling of Sus scrofa circular RNAs across nine organs and three developmental stages," DNA Research, vol. 24, no. 5, pp. 523-535, 2017.

[63] X. Wei, H. Li, J. Yang et al., "Circular RNA profiling reveals an abundant circLMO7 that regulates myoblasts differentiation and survival by sponging miR-378a-3p," Cell Death \& Disease, vol. 8, no. 10, pp. e3153-e3165, 2017.

[64] H. Ouyang, X. Chen, Z. Wang et al., "Circular RNAs are abundant and dynamically expressed during embryonic muscle development in chickens," DNA Research, vol. 25, no. 1, pp. 71-86, 2018.

[65] R. Yao, Y. Yao, C. Li et al., "Circ-HIPK3 plays an active role in regulating myoblast differentiation," International Journal of Biological Macromolecules, vol. 155, pp. 1432-1439, 2020.

[66] Y. Cao, S. You, Y. Yao et al., "Expression profiles of circular RNAs in sheep skeletal muscle," Asian-Australasian Journal of Animal Sciences, vol. 31, no. 10, pp. 1550-1557, 2018.

[67] S. Peng, C. Song, H. Li et al., "Circular RNA SNX29 sponges miR-744 to regulate proliferation and differentiation of myoblasts by activating the Wnt5a/Ca ${ }^{2+}$ signaling pathway," Molecular Therapy-Nucleic Acids, vol. 16, pp. 481-493, 2019.

[68] H. Li, J. Yang, X. Wei et al., "CircFUT10 reduces proliferation and facilitates differentiation of myoblasts by sponging miR133a," Journal of Cellular Physiology, vol. 233, no. 6, pp. 4643-4651, 2018.

[69] H. Li, X. Wei, J. Yang et al., "circFGFR4 promotes differentiation of myoblasts via binding miR-107 to relieve its inhibition of Wnt3a," Molecular Therapy - Nucleic Acids, vol. 11, pp. 272-283, 2018.
[70] P. Siengdee, N. Trakooljul, E. Murani, M. Schwerin, K. Wimmers, and S. Ponsuksili, "MicroRNAs regulate cellular ATP levels by targeting mitochondrial energy metabolism genes during C2C12 myoblast differentiation," PLoS One, vol. 10, no. 5, pp. e0127850-e0127869, 2015.

[71] Y. Wang, M. Li, Y. Wang et al., "A Zfp609 circular RNA regulates myoblast differentiation by sponging miR-194-5p," International Journal of Biological Macromolecules, vol. 121, pp. 1308-1313, 2019.

[72] A. Panda, K. Abdelmohsen, J. L. Martindale et al., "Novel RNA-binding activity of MYF5 enhancesCcnd1/Cyclin D1mRNA translation during myogenesis," Nucleic Acids Research, vol. 44, no. 5, pp. 2393-2408, 2016.

[73] P. Zhang, Z. Chao, R. Zhang et al., "Circular RNA regulation of myogenesis," Cell, vol. 8, no. 8, pp. 885-897, 2019.

[74] I. Legnini, G. di Timoteo, F. Rossi et al., "Circ-ZNF609 is a circular RNA that can be translated and functions in myogenesis," Molecular Cell, vol. 66, no. 1, pp. 22-37.e9, 2017.

[75] P. Pandey, J. Yang, D. Tsitsipatis et al., "circSamd4 represses myogenic transcriptional activity of PUR proteins," Nucleic Acids Research, vol. 48, no. 7, pp. 3789-3805, 2020.

[76] H. Yin, X. Shen, J. Zhao et al., "Circular RNA CircFAM188B encodes a protein that regulates proliferation and differentiation of chicken skeletal muscle satellite cells," Frontiers in Cell and Developmental Biology, vol. 8, pp. 1-15, 2020.

[77] Z. Chen, J. Zhou, M. Wang et al., "Circ09863 regulates unsaturated fatty acid metabolism by adsorbing miR-27a-3p in bovine mammary epithelial cells," Journal of Agricultural and Food Chemistry, vol. 68, no. 32, pp. 8589-8601, 2020.

[78] J. Sun, H. Zhang, B. Hu et al., "Emerging roles of heat-induced circRNAs related to lactogenesis in lactating sows," Frontiers in Genetics, vol. 10, pp. 1347-1347, 2020.

[79] X. Lin, J. Luo, L. Zhang, W. Wang, and D. Gou, "MiR-103 controls milk fat accumulation in goat (Capra hircus) mammary gland during lactation," PLoS One, vol. 8, no. 11, pp. e79258e79271, 2013.

[80] C. Dou, Z. Cao, B. Yang et al., "Changing expression profiles of lncRNAs, mRNAs, circRNAs and miRNAs during osteoclastogenesis," Scientific reports, vol. 6, no. 1, pp. 21499-21510, 2016.

[81] C. Zittle and J. Custer, "Purification and some of the properties of _ $\alpha_{-}$s-casein and _ $\kappa_{-}$-casein,” Journal of Dairy Science, vol. 46, no. 11, pp. 1183-1188, 1963.

[82] W. Jin, E. M. Ibeagha-Awemu, G. Liang, F. Beaudoin, X. Zhao, and L. Guan, "Transcriptome microRNA profiling of bovine mammary epithelial cells challenged with Escherichia coli or Staphylococcus aureus bacteria reveals pathogen directed microRNA expression profiles," BMC Genomics, vol. 15, no. 1, pp. 181-196, 2014.

[83] Y. Bian, Y. Lei, C. Wang et al., "Epigenetic regulation of miR29s affects the lactation activity of dairy cow mammary epithelial cells," Journal of Cellular Physiology, vol. 230, no. 9, pp. 2152-2163, 2015.

[84] J. Wright, "MicroRNAs and EMT in mammary cells and breast cancer," Journal of Mammary Gland Biology and Neoplasia, vol. 15, no. 2, pp. 213-223, 2010. 